\title{
Power-law Behavior of the Alternative Splicing of Exons in Human Transcriptome
}

\author{
Vasily V. Grinev ${ }^{1^{*}}$, Petr V. Nazarov ${ }^{2}$ and Eugene A. Klimov ${ }^{3,4}$ \\ ${ }^{1}$ Department of Genetics, Faculty of Biology, Belarusian State University, Minsk, Republic of Belarus. \\ ${ }^{2}$ Proteome and Genome Research Unit, Luxembourg Institute of Health, Strassen, Luxembourg. \\ ${ }^{3}$ Department of Genetics, Faculty of Biology, Lomonosov Moscow State University, Moscow, \\ Russian Federation. \\ ${ }^{4}$ Center of Experimental Embryology and Reproductive Biotechnologies, Moscow, \\ Russian Federation.
}

Authors' contributions

This work was carried out in collaboration among all authors. Author VVG designed the study, performed the analysis and wrote the first draft of the manuscript. Author PVN analyzed data. Author EAK wrote the final manuscript. All authors read and approved the final manuscript.

Article Information

DOI: 10.9734/ARRB/2019/v32i630105

Editor(s):

(1) Dr. Msafiri Yusuph Mkonda, Lecturer, Sokoine University of Agriculture, Tanzania. Reviewers:

(1) Michael Bordonaro, Geisinger Commonwealth School of Medicine, Pennsylvania, USA.

(2) Kiran Sharma, USA

Complete Peer review History: http://www.sdiarticle3.com/review-history/51032

Original Research Article

Received 22 June 2019

Accepted 27 August 2019

Published 02 September 2019

\section{ABSTRACT}

Aims: To establish the common rules of exon combinatorics during RNA splicing.

Study Design: Inferring a plausible statistical model of exon combinatorics from the annotated models of human genes during RNA splicing.

Place and Duration of Study: Department of Genetics (Belarusian State University), Proteome and Genome Research Unit (Luxembourg Institute of Health), Department of Genetics (Lomonosov Moscow State University) and Moscow Center of Experimental Embryology and Reproductive Biotechnologies, between January 2017 and July 2019.

Methodology: We used human mRNA and EST sequences from GenBank (1093522 unique records in total) and linear models of the human genes from Ensembl (58051 genes), AceView (72384 genes), ECgene (57172 genes), NCBI RefSeq (54262 genes), UCSC Genome Browser (58037 genes) and VEGA (54950 genes) to calculate a combinatorial index of human exons. We inferred the most plausible statistical model describing the distribution of combinatorial index of 
human exons using Clauset's mathematical formalism. Predictors of the combinatorial index values and functional outcomes of the predefined behavior of exons during splicing were also determined.

Results: Power-law is the most plausible statistical model describing the combinatorics of exons during RNA splicing. The combinatorial index of human exons is defined by more than $90 \%$ by the 138 features that have different importance. The most important of these features are the abundance of exon in transcripts, the strength of splice sites, the rank of exon in transcripts and the type of exon. Analysis of the marginal effects shows that different values of the same feature have unequal influence on the combinatorial index of human exons. Power-law behavior of exons during RNA splicing pre-determines structural diversity of transcripts, low sensitivity of splicing process to random perturbations and its high vulnerability to manipulation with highly combinative exons.

Conclusion: Exons widely involved in alternative splicing are a part of the common power-law phenomenon in human cells. The power-law behavior of exons during RNA splicing gives the unique characteristics to human genes.

Keywords: Human exons; RNA splicing; combinatorics; statistical modeling; power-law; predictors; functional outcomes.

\section{INTRODUCTION}

Alternative splicing is a unique process of unzipping genetic information archived in the nucleotide sequence of the gene. This is a widespread phenomenon in human cells and tissues. It was estimated that $92-94 \%$ of human genes produce appreciable levels of two or more distinct populations of RNA isoforms [1]. Alternative isoforms of transcripts may appear at the level of single cells or populations of cells of the same type [2,3], different tissues of the same individual or the same tissue but in different individuals $[1,4]$ and at different stages of human development $[5,6]$.

The main outcome of the alternative splicing is significant expansion of the complexity of the transcriptome, when a single gene can produce a wide variety of RNA molecules. These molecules may be translated into a variety of structurally and functionally distinct proteins [7]. Moreover, some of these molecules can be noncoding and may play a regulatory role [8]. A set of such diverse products of the same gene often forms a sub-network, which tightly integrates into the global cellular regulatory network and provides flexibility in cell function and adaptation $[9,10]$.

The implementation of high-throughput OMICStechnologies has substantially expanded our understanding of alternative splicing and its biological role. It also suggests that we see yet only the tip of the iceberg of the entire transcriptome complexity in a cell. The evergrowing set of empirical data in this area requires the elucidation of common principles or rules by which the transcriptome of a cell forms and functions. We hope that through the knowledge of such rules further progress in this area will be achieved. Over the last decade there have already been some successes in this direction. In particular, some basic properties of the "splicing code" were disclosed [11,12]. However, we still don't have full understanding of the rules of exon combinatorics and the systemic factors that drive and control the splicing process.

Analysis of the human transcriptome shows that the number of various splicing events involving exons may vary significantly for different exons. A large set of exons are only involved in a single splicing event. On the other hand, human transcriptome contains a limited number of exons, which take part in many different splicing events. In this regard, we set a goal to figure out whether there are principles of local combinatorics of exons and if so, where and how it is predefined.

In a context of this article, the term "local combinatorics of exons" refers to pairwise splicing events involving a given exon during formation of different RNA isoforms. In contrast, the term "extended combinatorics of exons" refers to splicing of different exons during formation of a given RNA isoform. For the purposes of this article, we also use the term "combinatorial properties of exons" which indicates a set of properties of exons which predetermine the diversity of their alternative splicing. Moreover, we introduced the "exon's combinatorial index" (ECl) which is an equivalent to a topological index "node degree" of graph theory. In a context of splicing, ECl indicates the number of unique splicing events in which a given exon is involved. In further analytical work we used both a total-degree of exon ("total" 
exon's combinatorial index or simply total-ECl) as well as its decomposed variant (separated ingoing and outgoing degrees, or in-ECl and out$\mathrm{ECl}$, respectively). Herewith, total- $\mathrm{ECl}$ indicates the sum of all the ingoing (forming of exon-exon junctions with upstream exons) and the outgoing (forming of exon-exon junctions with downstream exons) unique splicing events that involve an exon. The terms in- and out-ECl refer to the sum of all the ingoing or outgoing unique splicing events that involve an exon, respectively.

\section{MATERIALS AND METHODS}

For the purposes of this paper, human mRNA and ESTs sequences deposited in GenBank were downloaded via FTP-server of the UCSC Genome Browser. These sequences were aligned by BLAT [13] against GRCh38/hg38 reference assembly of the human genome and were subjected to four levels of filtration: records with only one aligned block, mismatches, exons and/or introns length below the $5^{\text {th }}$ quantile of distribution (23 and 88 nucleotides in length for exons and introns, respectively) were deleted. The resulting collection of sequences we called Dataset 1 with 1093522 records. Additionally, we trimmed terminal exons of sequences from Dataset 1 and formed Dataset 2 with 627733 records.

Moreover, for further validations of GenBankbased findings, linear models of the human genes from Ensembl (58051 genes, 546456 exons and 370720 exon-exon junctions), AceView (72384 genes, 629892 exons and 394462 exon-exon junctions), ECgene (57172 genes, 583433 exons and 393678 exon-exon junctions), NCBI RefSeq (54262 genes, 413114 exons and 165937 exon-exon junctions), UCSC Genome Browser (58037 genes, 546740 exons and 350863 exon-exon junctions) and VEGA (54950 genes, 587645 exons and 376591 exonexon junctions) were also downloaded as GTF/GFF files. All one-exon transcripts were removed from these annotations and data were converted into an object of a class TranscriptDb and saved as a local SQLite database.

Statistical modeling and statistical analysis of the above mentioned datasets was carried out using $R$ programming language. All customdeveloped $R$ codes used for the analysis are available upon request. The key steps of this analysis are described in the relevant sections of RESULTS.

\section{RESULTS AND DISCUSSION}

\subsection{RESULTS}

\subsubsection{Power-law behavior of local combinatorics of human exons}

Our work is based on the analysis of seven data sets: Full list of human mRNA and EST sequences from GenBank [14] and linear models of the human genes from Ensembl [15], AceView [16], ECgene [17], NCBI RefSeq [18], UCSC Genome Browser [13] and VEGA [19]. For a more compact representation and future use, these data were converted into exon graphs. Each of exon graphs is presented by a set of exons (vertices or nodes of a graph) connected to each other via a set of splicing events (edges or links of a graph) [20]. Such a graph is a directed acyclic graph in a sense that the exons present in any mature transcript of a gene are retained in the correct 5' to 3' linear order and the reverse edges are prohibited.

The results of the topological analysis of reconstructed exon graphs suggest that the $\mathrm{ECI}$ values follow a power-law distribution with a heavy right tail: the vast majority of exons have low $\mathrm{ECl}$ value, while a small proportion of the exons are characterized by a very high $\mathrm{ECl}$ value (Fig. 1A). However, power-law is only one of the members of a broad family of distributions with heavy right tails [21]. In addition, the selection of the correct statistical model for that kind of data is not a trivial task because of the incompleteness of the empirical biological data and their high variability (especially in the area of the heavy tail). Therefore, we had to use a threestep approach based on the mathematical formalism developed by Clauset $A$. et al. [22,23] to find an appropriate statistical model and to check our preliminary hypothesis.

First, we rejected those statistical models that clearly did not fit the empirical distributions and chose five closest models: Power-law distribution, truncated power-law distribution (or power-law with exponential cut-off), exponential distribution, stretched exponential distribution (or complementary cumulative Weibull distribution) and log-normal distribution. Next, selected statistical models were fitted to the empirical distributions according to " $x_{\text {min }}$ " paradigm [22]: only heavy tail of the empirical distribution was the subject of our attention because it contains the most outstanding sub-set of values of the distribution. Finally, Kolmogorov-Smirnov test 
and log-likelihood ratio test were used to assess the plausibility of the statistical hypothesis and to directly compare the alternative statistical models $[22,24,25]$.

The above-mentioned approach allowed us to identify several features of empirical distributions. First, the results of our statistical modeling allow to postulate that the $\mathrm{ECl}$ values of human exons follow a truncated form of the power-law with an exponential cut-off (Fig. 1B, Table 1). Herewith, an exponential component of the distribution can be substantially reduced by filtering out the 5'and 3'-terminal exons (with in-ECl $=0$ and out$\mathrm{ECl}=0$, respectively) together with the edges and first neighbours from exon graphs (data not shown). However, it should be noted that for the three data sets (gene models from AceView, NCBI RefSeq and VEGA) there remains uncertainty when truncated power-law is compared with stretched exponential or lognormal models: log-likelihood ratio test does not favor one model over the other and only Kolmogorov-Smirnov test gives a slight preference for the power-law with exponential cut-off. Second, the beginning of the heavy tail (lower bound) for different data sets ranges from 5 to 15. Third, in the frame of a truncated powerlaw model the scaling parameter $\alpha$ lies within the range from 2.378 to 7.248 and rate parameter $\lambda$ falls into the broad range from $5.713 \times 10^{-9}$ to $1.77 \times 10^{-1}$ for different data sets.
A
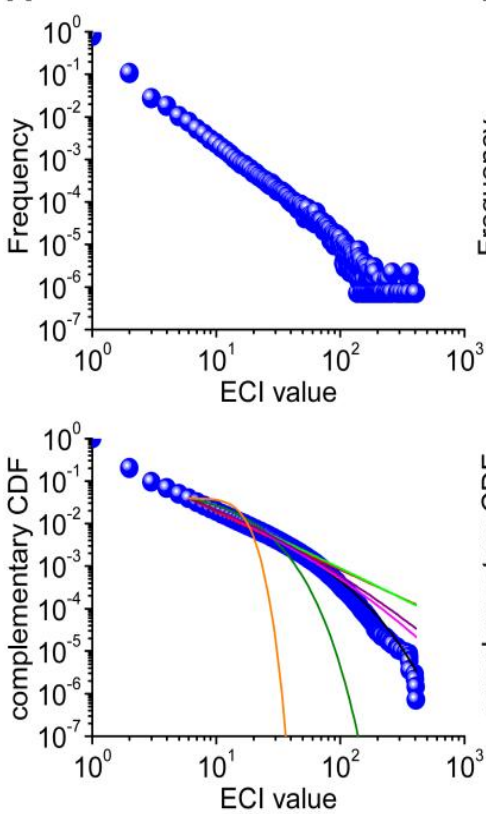

B

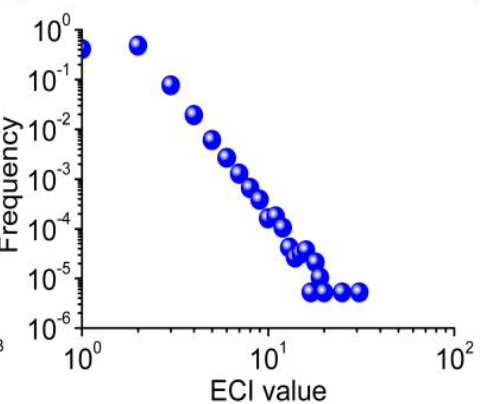

C

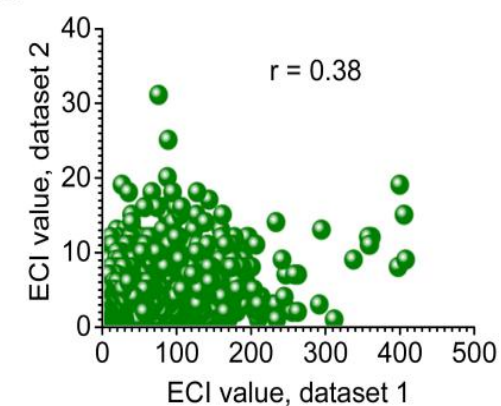

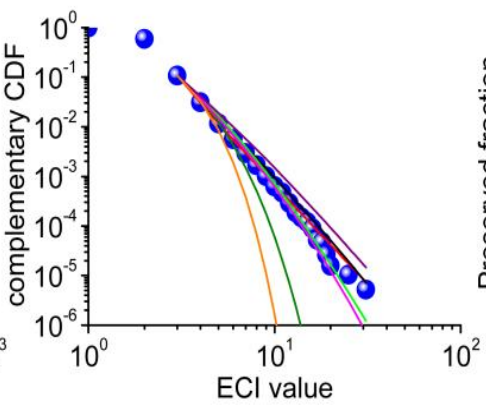

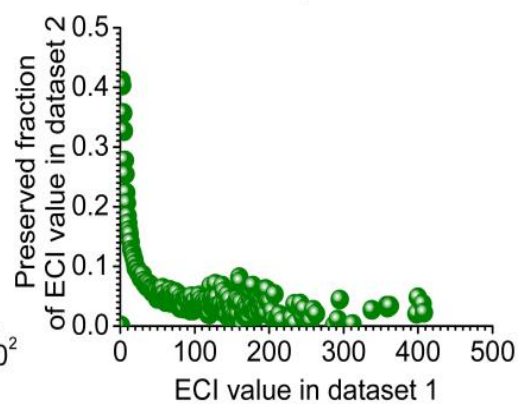

Legend to $(A)$ and $(B)$ :

- empirical data - truncated power-law - exponential $\quad$ - log-normal

- power-law - Yule-Simon - stretched exponential - Poison

Fig. 1. Removal of uncertainty by filtering out the terminal exons leads to a clear manifestation of the power-law component in the human transcriptome

(A) Frequency (upper panel) and complementary CDF (lower panel) plots of the ECI values distribution from the whole set of human transcripts and exons. Truncated power-law with an exponential cut-off is the best statistical model for this empirical distribution among the set of competitive models of distributions with heavy right tail; (B) Frequency (upper panel) and complementary CDF (lower panel) plots of the $\mathrm{ECl}$ values distribution after removal of the terminal exons from transcripts and after data reanalysis. For these transformed data, there exists a clear superiority of the power-law model as compared to other statistical models; (C) Removal of the terminal exons from transcripts leads to significant change in the ECl values of exons (upper panel). Herewith, the exons with initially high values of the $\mathrm{ECl}$ underwent the most profound changes (lower panel) 
Table 1. Log-likelihood ratio test (A) and statistical tests on plausibility (B) confirm the presence of a power-law component in the human transcriptome

\begin{tabular}{|c|c|c|c|c|c|c|c|c|c|}
\hline & & & & & $\mathbf{A}$ & & & & \\
\hline Dataset & Basic model & LLR & & & Com & eting statistic & I model & & \\
\hline & & test & Power-law & $\begin{array}{l}\text { Truncated } \\
\text { power-law }\end{array}$ & Yule-Simon & Exponential & $\begin{array}{l}\text { Stretched } \\
\text { exponential }\end{array}$ & Log-normal & Poison \\
\hline Dataset 1 & Power-law & $\mathrm{R}$ & - & -446.6 & -20.6 & 41.22 & -17.7 & -19.6 & 51.03 \\
\hline & & $\mathrm{p}$ & - & $3.0 e-196$ & 2.3e-94 & 0.0 & $2.8 e-70$ & $3.5 e-85$ & 0.0 \\
\hline & Truncated & $\mathrm{R}$ & 446.6 & - & 30.5 & 47.34 & 20.6 & 30.9 & 51.23 \\
\hline & power-law & $\mathrm{p}$ & 1 & - & 0.0 & 0.0 & 0.0 & 0.0 & 0.0 \\
\hline Dataset 2 & Power-law & $\mathrm{R}$ & - & -0.8 & 4.2 & 11.12 & 3.6 & -0.6 & 13.64 \\
\hline & & $p$ & - & 0.2 & $2.3 e-05$ & 0.0 & $3.4 \mathrm{e}-04$ & 0.5 & 0.0 \\
\hline & Truncated & $\mathrm{R}$ & 0.8 & - & 4.8 & 11.37 & 4.0 & 0.8 & 13.76 \\
\hline & power-law & $p$ & 1 & - & $2.0 \mathrm{e}-06$ & 0.0 & $5.2 e-05$ & 0.4 & 0.0 \\
\hline & & & & & B & & & & \\
\hline Dataset & Test & & & & Compe & ng statistical & odel & & \\
\hline & & & er-law & $\begin{array}{l}\text { Truncated } \\
\text { power-law }\end{array}$ & Yule-simon & ponential & $\begin{array}{l}\text { Stretched } \\
\text { exponential }\end{array}$ & Log-normal & Poison \\
\hline Dataset 1 & $\mathrm{AIC}$ & & 05.6 & 344314.5 & 345304.3 & 3155.6 & 344595.3 & 344593.7 & 897718.6 \\
\hline & $\mathrm{BIC}$ & & 14.5 & 344332.3 & 345313.2 & 3164.5 & 344613.1 & 344611.5 & 897727.5 \\
\hline & KS distance & & 715 & 0.02909 & 0.01553 & 7865 & 0.03159 & 0.03015 & 0.23341 \\
\hline Dataset 2 & $\mathrm{AIC}$ & & 4.8 & 39065.2 & 39158.1 & 201.8 & 39140.2 & 39065.6 & 42408.7 \\
\hline & $\mathrm{BIC}$ & & 2.7 & 39081.1 & 39166.0 & 209.7 & 39156.1 & 39081.4 & 42416.6 \\
\hline & KS distance & & 188 & 0.04049 & 0.01658 & 5476 & 0.04989 & 0.05232 & 0.08236 \\
\hline
\end{tabular}


It should be noted that the observed empirical distributions with power-law component cannot be produced by random attachment of exons during the splicing step of gene expression (Fig. 1C). On the other hand, this class of distributions can be easily generated by a preferential attachment process [26]. In a frame of preferential attachment model, different exons have different attractiveness to connect to other exons. The results of our modeling indicate that artificial data can be fitted to any of our empirical data set by varying the power parameter a (Fig. 1C). However, the exact nature of the observed difference in exon attractiveness has not been considered. This question is investigated below.

\subsubsection{Predictors of the value of $\mathrm{ECls}$ in human transcriptome}

It seems to be true that almost all (if not all) processes in the cell are controlled by complex multilevel mechanisms [27,28] and splicing process is unlikely to be an exception. So, we hypothesized that the value of $\mathrm{ECl}$ is determined by multiple predictors. To find those, we assembled a compendium of 22114 features of the target exons (ECls of which are the objects of analysis) and their upstream and downstream first neighboring exons and adjacent introns. This compendium included seven classes of features: abundance of exons in transcripts, connectivity of first neighboring exons, sequence features of the target exons, sequence features of the first neighboring exons, length of the adjacent introns, rank of the exons in transcripts and functional type of the target exons (Fig. 2). We took the Ensembl data set as the main object of research, and the other sets of data were used for crossvalidation of the results. The relationship between a feature and an $\mathrm{ECl}$ as well as contribution of the feature to the value of the $\mathrm{ECI}$ was assessed by the pairwise Spearman's rank correlation coefficient and by data mining. Data mining was based on machine learning by regression random forests. This learning algorithm was chosen by comparison with the two other algorithms (lasso regression and generalized boosting regression) as the most suitable for the task.

A

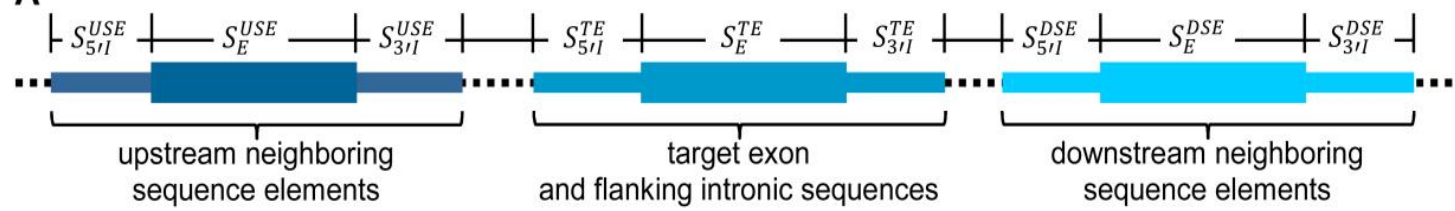

B

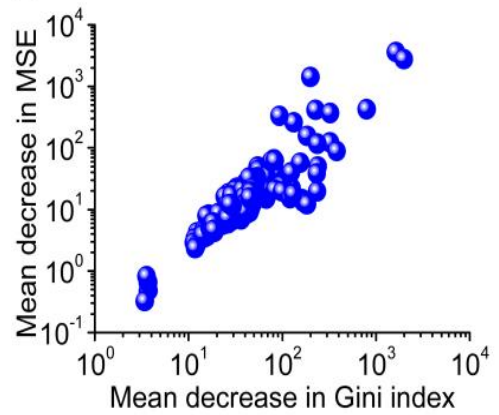

C

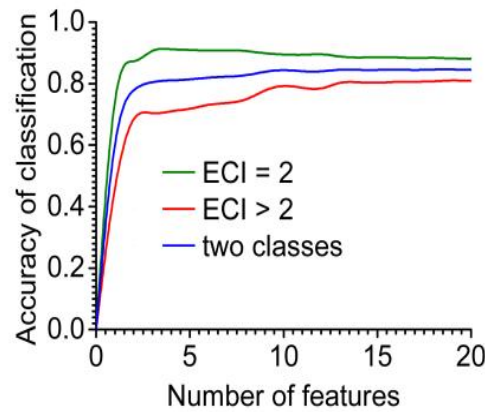

D

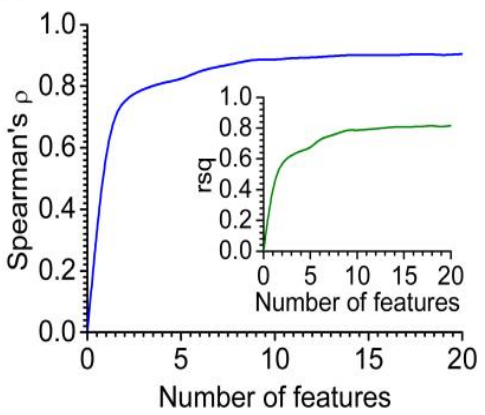

Fig. 2. Small subset of features from exons and flanking introns can determine the value of the $\mathrm{ECl}$ in tissue-independent fashion

(A) Features of the five different classes (sequence features, sequence-related features, functional features, epigenetic features and features related to structure of a gene) were extracted from three classes of the genomic/RNA elements: Target exon and its flanking intronic sequences, upstream first neighboring exons and their flanking intronic sequences and downstream first neighboring exons and corresponding flanking intronic sequences; (B) Importance of these features for the prediction of the ECI value was inferred from random forest classification by Gini index and from random forest-based multiple nonlinear regression by mean square error;

(C) Random forest-based classification shows that the maximum accuracy in prediction of the $\mathrm{ECl}$ is achieved by using no more than top-20 features; (D) Top-20 features allow to achieve a maximum in explaining the $\mathrm{ECI}$ variance and high accuracy in prediction of the $\mathrm{ECl}$ values by random forest-based multiple nonlinear regression 
We found that for any gene of interest the presence of multiple exons, a wide spectrum of produced transcripts and different abundance of exons in these transcripts are minimal prerequisites for non-equivalency of the $\mathrm{ECl}$ value. The Most interesting was the effect of the abundance of exons in transcripts. On one hand, there is a high positive correlation between the value of ECls and the abundance of target exons in transcripts, and data mining revealed high importance of this predictor. However, the converse statement is not true: Not all exons widely represented in transcripts are characterized by high combinatorial capacity. Moreover, profiles of the marginal effects point out to the unequal importance of the different values of this feature in the determination of the $\mathrm{ECl}$. On the other hand, the abundance itself is determined by multiple factors as was shown early [11].

The Second class of features (connectivity of first neighboring exons) has a moderate or little effect on the value of $\mathrm{ECl}$ as revealed by correlation analysis and data mining by random forests. In general, exon graphs are disassortative (with value of disassortativity index up to -0.247 ): the exons with high values of $\mathrm{ECl}$ prefer to attach to the exons with low value and vice versa.

In our compendium, classes sequence features of target and first neighboring exons include length of exons, linear density of minimal free energy of exons, strength of the 5' and 3' splice sites, regional counts of the short motifs (1-3 nucleotides), frequency of the known splicing enhancer and silencer motifs and count of the new predicted motifs associated with high combinatorial exons (MAHCE). One of our approaches in identification of MAHCE was correlation-based approach. This approach allowed us to cluster many sequences while discarding irrelevant oligomers. In turn, the decrease in the number of the unique sequences led to a reduction in the dimension of the space of variables and allowed to use the machine learning algorithms for the identification of important predictors.

Despite the large number of studied structural features as well as cis-elements, and carefully carried out analysis we didn't find strong predictors. The most significant was the relationship between the strength of the splice sites and the value of the ECl. It should be noted that there is a clear cross-relationship between the value of the in- or out-ECl and the strength of the respective splice site as well as increased correlation at use of the total (overall) score of the strength of splice sites. Herewith, the profiles of marginal effects of the splice site strength of the target and first neighboring exons are completely different. The remaining cis-elements have a moderate or little effect on the value of $\mathrm{ECl}$ (both the total-ECl and the in- and out-ECl) as was revealed by correlation analysis and data mining by random forests. A similar situation was observed such parameters such as length of exons and stability of their secondary structure.

From next class of features, we studied the minimal, maximal and mean length of the adjacent introns in relation to the $\mathrm{ECl}$ value. These predictors were successfully selected by feature selection algorithm as important for prediction of the $\mathrm{ECl}$, but with low contribution (no more than $9.33 \%$ average increase in squared out-of-bag residuals), which agrees with results of correlation analysis.

One of the most informative and important features in prediction of the value of $\mathrm{ECl}$ was the position of exon in transcripts. We used two different approaches to determine this metric: averaged short (total-, in- and/or out-) distance of exon to another exons in exon graph and direct determination of averaged exon position (exon rank) relative to the start (5'-end) and/or to the end (3'-end) in the transcripts of the gene of interest. Simple ratio of these two parameters gives us the position of the exon relative to the center of transcripts (the centrality of exon position in transcripts). The centrality of exon position was expressed either in relative units (where 1 is a relative center of transcripts, which include the exon of interest) or in absolute distance (measured in the number of exons) from the center of transcripts. In the latter case, zero position indicates the center of transcripts which include the exon of interest. If exon is located to the left of the center (closer to the 5'-end of transcripts), its position has a negative sign, otherwise a positive sign.

All exons with high values of total-ECl show nonrandom positional distribution and tend to occupy central position in transcripts (Fig. 3). Permutation of the values of predictors from this class of features increases more than $30 \%$ of squared out-of-bag residuals in a case of target exons, and more than $20 \%$ in a case of first neighboring exons. Again, as was mentioned for other features, profiles of the marginal effects point out to the unequal importance of the 
different values of this predictor in the determination of the $\mathrm{ECl}$ with clear transition point near the central position.

Finally, we studied a possible influence of evolutionary conservatism of exon and its functional type on the value of the $\mathrm{ECl}$. We found that a group of exons with high values of ECls $(\geq 10)$ is characterized by an average level of conservatism. However, we observed a low positive correlation between the conservatism of exon and the value of its $\mathrm{ECl}$ and, thus, this variable was not selected as important by feature selection algorithm. As for functional type of exon, ANOVA and data mining by random forests confirm the importance of this feature in determination of the value of $\mathrm{ECl}$. In particular, there is a clear link between the multifunctionality (when exon is annotated as a multitype exon) of exon and high value of $\mathrm{ECl}$, and permutation of the values of this feature increases more than $30 \%$ of squared out-of-bag residuals.

In summary, as it was originally supposed, among the features we studied there was no single predictor or a small group of predictors that would entirely determine the value of the $\mathrm{ECI}$ of human exons. On the contrary, the value of the $\mathrm{ECl}$ is defined more than $90 \%$ by the multidimensional space of predictors (138 features in a case of total-ECl of Ensembl exons) that have different importance. The most important of these predictors are abundance of exon in transcripts, strength of splice sites, rank of exon in transcripts and type of exon. Furthermore, analysis of the marginal effects shows that even different values of the same predictor have an unequal influence on the $\mathrm{ECl}$.

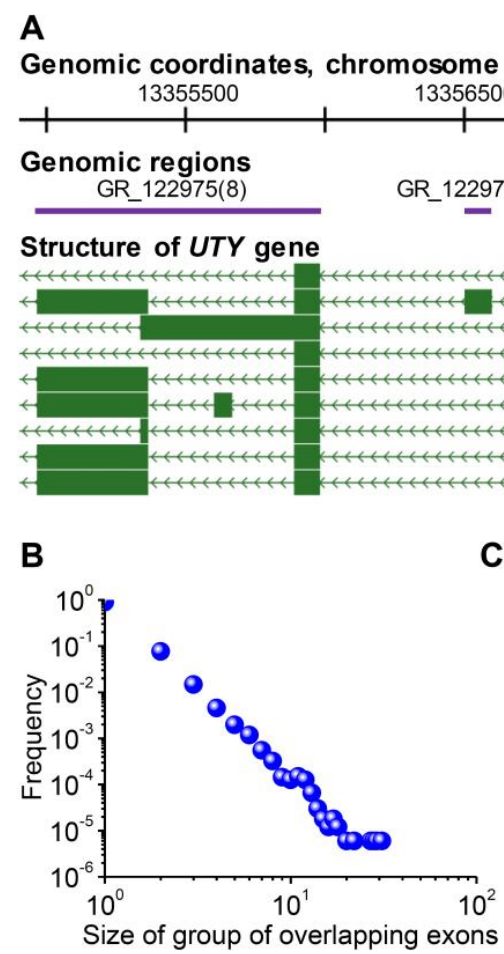

C

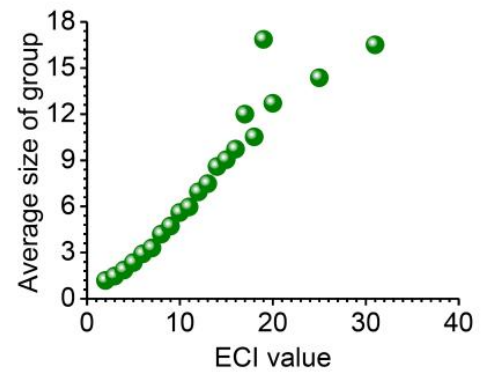

D

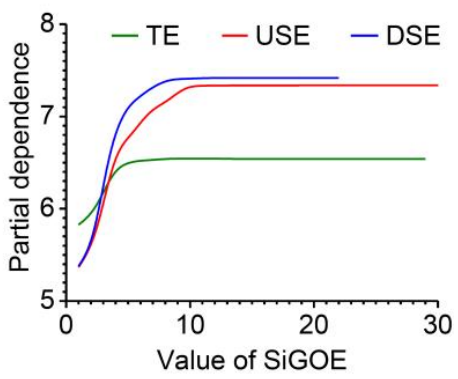

Fig. 3. The most important predictor of the $\mathrm{ECl}$ value of the target exon is a structure of its upstream and/or downstream neighboring exon-coding genomic regions

(A) Genomic structure of the small part of UTY gene is used as example. Exon-coding genomic regions that belong to this part of the gene are depicted as GR. The number of exons originated from each genomic region (size of group of overlapping exons, or SiGOE) is indicated in parentheses. Target exon is colored in dark blue.

This exon has $\mathrm{ECl}=10$ and $70 \%$ of its splicing events happening with exons from two genomic regions

GR_122975 and GR_122978; (B) Empirical distribution of sizes of groups of overlapping exons from human genome follows a power-law function; (C) The relationship between the $\mathrm{ECl}$ value of target exon and the average size of its neighboring upstream and/or downstream groups of overlapping exons. This relationship is close to linear; (D) Marginal effects of size of neighboring upstream (USE) and downstream (DSE) groups of overlapping exons on the ECl value of the target exon. The effect of size of overlapping group that owns the exon (TE) is also shown 


\subsubsection{Exon graphs with power-law structure: The functional outcomes}

To establish the biological significance of powerlaw structure of the exon graphs, we compared this type of graphs with artificial full exon graphs based on three criteria: Diversity of the generated transcripts, flexibility of the alternative splicing and robustness to the random perturbations. In our modeling, we used sub-set of top-100 exon graphs (in terms of the number of vertices) from Ensembl-based human transcriptome exon graph. These empirical exon graphs (which we called power-law exon graphs) have topology with power-law component while our artificial full exon graphs do not have this component in the distribution of the $\mathrm{ECl}$.

As was expected, full exon graphs are capable of producing significantly more diverse transcripts than exon graphs with power-law component. For example, directed walk along the tree of exon graphs shows that full exon graphs generate 6.6 fold more different transcripts than power-law exon graphs and 210.4-fold more than it was experimentally verified $\left(p=1 \times 10^{-16}\right)$. However, the length of such transcripts is clearly smaller than the length of transcripts generated by power-law exon graphs as well as empirical transcripts. In addition, a full crawl of the powerlaw exon graphs shows that they have great hidden potential to generate a variety of transcripts, a superior variety to known transcripts (1472.2-fold). Herewith, this potential can be seen not only in the structural diversity, but in the ability to generate long transcripts: there are clearly two distant peaks compared to the empirical data.

Next, of interest was a flexibility of the alternative splicing with different types of exon graphs. We modeled the situation when any fraction of the ranked exons was purposefully skipped or included in the mature transcripts. Power-law exon graphs are extremely sensitive to manipulation with top-ranked exons: active inclusion or skipping even of a small fraction of these exons into splicing process may substantially change the possibilities for the formation of a variety of transcripts. At the same time, full exon graphs do not have such flexibility. This applies to both the structural diversity of transcripts, and a variety of lengths of transcripts.

Finally, we modeled the effect of random perturbations on the different types of exon graphs and tested their ability to withstand such perturbations. Exact physical nature of random perturbations may be different, for example, it can be an accidental loss of exon(-s) because of the deletion at genomic DNA level or failure to include exon(-s) into mature RNA because of mutations of the splicing cis-regulatory elements. The results of our modeling indicate that full exon graphs are significantly more robust to random factors than power-law exon graphs. This difference is most clearly seen in the case where the robustness is estimated to change the length of the generated transcripts.

\section{DISCUSSION}

Power-law distributions appear in an enormous variety of fundamentally different complex systems: from engineering to biological and social systems [29,21]. Biological systems as the most complex systems are particularly rich in this phenomenon which is manifested at all levels of the organization of living organisms, from the molecular to the ecosystem level [30,29,31]. Therefore, it is not surprising that the combinatorial properties of human exons are subject to the same law. Why this phenomenon is so common in biological systems? Perhaps this is due the unique properties the system acquires, when the distribution of some of its parameters obeys power-law.

The first of these properties is scale-free distribution [21]. We have observed this property in our data sets. For example, random sampling of GenBank data didn't change the ratio between the numbers of exons with high and low values of $\mathrm{ECl}$ or randomly sampled sub-set of Ensembl data didn't change the form of distribution (data not shown). The main outcome of scale-free is the scalability of the system without losing its characteristics. So, we may speculate that power-law component allows to adaptively scale up or scale down the transcriptome in individual human cells in response to environmental conditions without changing the critical system parameters.

The second property of the systems with powerlaw component is adaptive flexibility. It was shown in the model and experimental studies that power-law distribution of any systemic parameter is a sign that the system is in the vicinity of phase transition or critical point [21,32]. Being close to the critical point, the system can be quickly reconstructed and can adapt to changing environmental conditions [33]. Our results are consistent with these ideas. In 
particular, our modeling shows that actively involving into splicing process or skipping even of a small fraction of the exons with high value of $\mathrm{ECl}$ may substantially change the possibilities for the formation of a variety of transcripts. Moreover, the theoretically possible diversity calculated on the basis of the structure of exon graphs is much greater than the experimentally confirmed diversity of the transcripts in human transcriptome. Consequently, human genes have significant hidden potential to produce different RNA molecules. Of course, it may be also due to incompleteness of empirical data or the existence of obscure limits on the formation of some variants of the transcripts, and this can be the topic of a separate study.

The third property of systems with power-law component is their sensitivity to accidental damages. Despite the fact that the complex biological systems can stably operate in various conditions, they yet are fragile [34]. We see this in our model studies, the results of which show that the exon graphs are sensitive to random perturbations. To improve the robustness of such fragile living systems, the nature has taken the path of increasing diversity and complexity of regulatory mechanisms $[27,28]$. Splicing process is controlled by a variety of mechanisms based on a redundant inner diversity of the cis- and trans-regulatory factors. These factors are organized in a spatially and functionally distributed intracellular network with multiple positive and negative forward and feedback reverse regulatory circuits (Braunschweig $U$. et al., 2013). We believe that because of this, we could not find one or more predictors which would completely determine the value of the $\mathrm{ECl}$. Instead of that, we found more than one hundred features that are involved in determining the value of $\mathrm{ECl}$. A similar situation exists with other properties of splicing, for example, more than two hundreds predictors that determine the inclusion or exclusion of exon in/from the mature transcripts were identified in different types of human tissues [11].

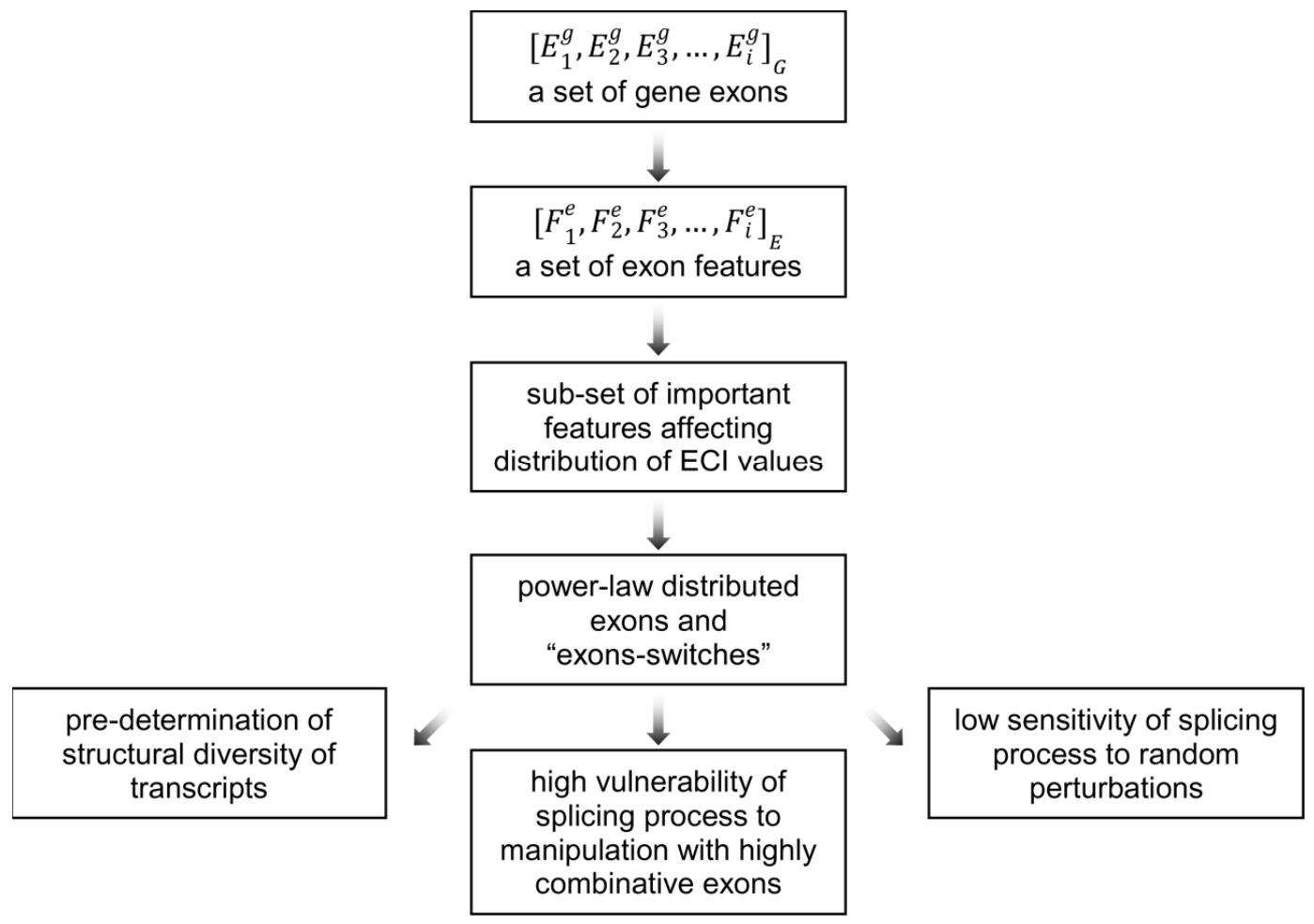

Fig. 4. Power-law phenomena in RNA splicing

Each exon of multi-exon genes can be characterized by a set of splicing-related features. Some of these features affect the normal distribution of ECI values and lead to the formation of a power-law distribution. The extreme manifestation of the power-law distribution is the appearance of "exons-switches". In addition, the power-law component gives specific properties to the human genes and splicing process: it pre-determines structural diversity of transcripts of a gene, low sensitivity of splicing process to random perturbations and its high vulnerability to manipulation with highly combinative exons 
In light of the problem of predictors, the most interesting and unexpected for the $\mathrm{ECl}$ were such features as the position of the exon in transcripts and the functional type of exon. In fact, the high-combinatorial exons are not only "hot points" of alternative splicing but they prefer to be located near the alternative transcription start and termination sites (however these exons usually are not 5'- or 3'-terminal exons). Exon 8b of human RUNX1T1 gene is a typical example. And our metric "centrality of exon position in transcripts" reflects only average position of exon in transcripts that include the exon of interest. However, this feature is highly informative. Moreover, we also believe that highly combinative exons due to the specificity of their location are usually multifunctional (these exons can be 5'UTR, 5'UTR/CDS, CDS, CDS/3'UTR and/or 3'UTR exon depends on transcript) and are characterized by a middle level of conservatism.

\section{CONCLUSION}

In summary, our results confirm the existence of the "exons-switches" of alternative splicing [1]. However, we have made substantial refinements in this concept. In particular, we showed that the "exons-switches" are part of the common power-law phenomenon in human cells. We also found that the combinatorial properties of human exons are defined by more than $90 \%$ by the multidimensional space of predictors that have different importance and different profiles of the marginal effects. Finally, we found that the power-law component gives the unique characteristics to the human genes (Fig. 4).

\section{ACKNOWLEDGEMENTS}

This work was supported in part by the Ministry of Education of the Republic of Belarus, grant \#3.08.3 (947/54, 469/54).

\section{COMPETING INTERESTS}

Authors have declared that no competing interests exist.

\section{REFERENCES}

1. Wang ET, Sandberg R, Luo $S$, Khrebtukova I, Zhang L, Mayr C, Kingsmore SF, Schroth GP, Burge CB. Alternative isoform regulation in human tissue transcriptomes. Nature. 2008; 456(7221):470-476.

DOI: $10.1038 /$ nature07509

2. Gurskaya NG, Staroverov DB, Zhang L, Fradkov AF, Markina NM, Pereverzev AP, Lukyanov KA. Analysis of alternative splicing of cassette exons at single-cell level using two fluorescent proteins. Nucleic Acids Research. 2012;40(8):e57.

DOI: 10.1093/nar/gkr1314

3. Marinov GK, Williams BA, McCue K, Schroth GP, Gertz J, Myers RM, Wold BJ. From single-cell to cell-pool transcriptomes: Stochasticity in gene expression and RNA splicing. Genome Research. 2014;24(3):496-510.

DOI: 10.1101/gr.161034.113

4. Castle JC, Zhang C, Shah JK, Kulkarni AV, Kalsotra A, Cooper TA, Johnson JM. Expression of 24,426 human alternative splicing events and predicted cis regulation in 48 tissues and cell lines. Nature Genetics. 2008;40(12):1416-1425.

DOI: $10.1038 /$ ng.264

5. Gamazon ER, Stranger BE. Genomics of alternative splicing: Evolution, development and pathophysiology. Human Genetics. 2014;133(6):679-687.

DOI: 10.1007/s00439-013-1411-3

6. Mazin $\mathrm{P}$, Xiong J, Liu X, Yan Z, Zhang $X$, Li M, He L, Somel M, Yuan Y, Phoebe Chen YP, Li N, Hu Y, Fu N, Ning Z, Zeng $\mathrm{R}$, Yang H, Chen W, Gelfand M, Khaitovich $P$. Widespread splicing changes in human brain development and aging. Molecular Systems Biology. 2013;9:633.

DOI: $10.1038 / \mathrm{msb} .2012 .67$

7. Nilsen TW, Graveley BR. Expansion of the eukaryotic proteome by alternative splicing. Nature. 2010;463(7280):457-463.

DOI: $10.1038 /$ nature08909

8. Rosa A, Brivanlou AH. Regulatory noncoding RNAs in pluripotent stem cells. International Journal of Molecular Sciences. 2013;14(7):14346-14373.

DOI: 10.3390/ijms140714346

9. Biamonti G, Caceres JF. Cellular stress and RNA splicing. Trends in Biochemical Sciences. 2009;34(3):146-153.

DOI: 10.1016/j.tibs.2008.11.004

10. Chandler DS, Singh RK, Caldwell LC, Bitler JL, Lozano G. Genotoxic stress induces coordinately regulated alternative splicing of the p53 modulators MDM2 and MDM4. Cancer Research. 2006;66(19): 9502-9508.

DOI: 10.1158/0008-5472.CAN-05-4271 
11. Barash $Y$, Calarco JA, Gao W, Pan Q, Wang $X$, Shai $O$, Blencowe BJ, Frey BJ. Deciphering the splicing code. Nature. 2010;465(7294):53-59.

DOI: 10.1038/nature09000

12. Matlin AJ, Clark F, Smith CW. Understanding alternative splicing: Towards a cellular code. Nature Reviews Molecular Cell Biology. 2005;6(5):386398.

DOI: $10.1038 / \mathrm{nrm} 1645$

13. Karolchik D, Barber GP, Casper J, Clawson $H$, Cline MS, Diekhans $M$, Dreszer TR, Fujita PA, Guruvadoo L, Haeussler M, Harte RA, Heitner $S$, Hinrichs AS, Learned K, Lee BT, Li CH, Raney BJ, Rhead B, Rosenbloom KR, Sloan CA, Speir ML, Zweig AS, Haussler D, Kuhn RM, Kent WJ. The UCSC Genome Browser database: 2014 update. Nucleic Acids Research. 2014; 42(Database issue):D764-770.

DOI: $10.1093 /$ nar/gkt1168

14. Benson DA, Clark K, Karsch-Mizrachi I, Lipman DJ, Ostell J, Sayers EW. Gen Bank. Nucleic acids research. 2014; 42(Database issue):D32-37.

DOI: $10.1093 /$ nar/gkt1030

15. Flicek $P$, Amode MR, Barrell D, Beal K, Billis $K$, Brent $S$, Carvalho-Silva $D$, Clapham P, Coates G, Fitzgerald S, Gil L, Giron CG, Gordon L, Hourlier T, Hunt S, Johnson $\mathrm{N}$, Juettemann $\mathrm{T}$, Kahari AK, Keenan S, Kulesha E, Martin FJ, Maurel T, McLaren WM, Murphy DN, Nag R, Overduin B, Pignatelli M, Pritchard B, Pritchard E, Riat HS, Ruffier M, Sheppard D, Taylor K, Thormann A, Trevanion SJ, Vullo A, Wilder SP, Wilson M, Zadissa A, Aken BL, Birney $E$, Cunningham F, Harrow $J$, Herrero J, Hubbard TJ, Kinsella R, Muffato M, Parker A, Spudich G, Yates A, Zerbino DR, Searle SM. Ensembl 2014. Nucleic Acids Research. 2014; 42(Database issue):D749-755.

DOI: 10.1093/nar/gkt1196

16. Thierry-Mieg D, Thierry-Mieg J. AceView: A comprehensive cDNA-supported gene and transcripts annotation. Genome Biology. 2006;7(Suppl 1):11-14.

DOI: $10.1186 / g b-2006-7-s 1-s 12$

17. Kim $P, \operatorname{Kim} N$, Lee $Y$, Kim B, Shin Y, Lee S. ECgene: Genome annotation for alterna-tive splicing. Nucleic Acids Research. 2005;33(Database issue):D7579.

DOI: $10.1093 /$ nar/gki118
18. Pruitt KD, Brown GR, Hiatt SM, ThibaudNissen $F$, Astashyn A, Ermolaeva $O$, Farrell CM, Hart J, Landrum MJ, McGarvey KM, Murphy MR, O'Leary NA, Pujar S, Rajput B, Rangwala SH, Riddick LD, Shkeda A, Sun H, Tamez P, Tully RE, Wallin C, Webb D, Weber J, Wu W, DiCuccio M, Kitts P, Maglott DR, Murphy TD, Ostell JM. RefSeq: An update on mammalian reference sequences. Nucleic Acids Research. 2014;42(Database issue): D756-763.

DOI: $10.1093 /$ nar/gkt1114

19. Harrow JL, Steward CA, Frankish A, Gilbert JG, Gonzalez JM, Loveland JE, Mudge J, Sheppard D, Thomas M, Trevanion S, Wilming LG. The vertebrate genome annotation browser 10 years on. Nucleic Acids Research. 2014; 42(Database issue):D771-779.

DOI: 10.1093/nar/gkt1241

20. Heber S, Alekseyev M, Sze SH, Tang H, Pevzner PA. Splicing graphs and EST assembly problem. Bioinformatics. 2002; 18(Suppl 1):S181-188.

DOI: 10.1093/bioinformatics/18.suppl_1.s181

21. Newman MEJ. Power laws, pareto distributions and Zipf's law. Contemporary Physics. 2005;46(5):323-351. DOI: $10.1080 / 00107510500052444$

22. Clauset A, Shalizi CR, Newman MEJ. Power-law distributions in empirical data. SIAM Review. 2009;51(4):661-703. DOI: $10.1137 / 070710111$

23. Virkar Y, Clauset A. Power-law distributions in binned empirical data. The Annals of Applied Statistics. 2014;8(1):89119.

DOI: 10.1214/13-aoas710

24. Klaus A, Yu S, Plenz D. Statistical analyses support power law distributions found in neuronal avalanches. PloS One. 2011;6(5):e19779.

DOI: 10.1371/journal.pone.0019779

25. Vuong $\mathrm{QH}$. Likelihood ratio tests for model selection and non-nested hypotheses. Econometrica. 1989;57(2):307. DOI: $10.2307 / 1912557$

26. Albert $\mathrm{R}$, Barabási AL. Statistical mechanics of complex networks. Reviews of Modern Physics. 2002;74(1):47-97. DOI: 10.1103/RevModPhys.74.47

27. Grinev VV, Ramanouskaya TV, Gloushen SV. Multidimensional control of cell structural robustness. Cell Biology International. 2013;37(10):1023-1037. DOI: $10.1002 /$ cbin. 10128 
28. Stelling J, Sauer U, Szallasi Z, Doyle FJ, 3rd, Doyle J. Robustness of cellular functions. Cell. 2004;118(6):675-685. DOI: 10.1016/j.cell.2004.09.008

29. Koonin EV, Wolf YI, Karev GP. Power laws, scale-free networks and genome biology. Molecular biology intelligence unit. Landes Bioscience/Eurekah.com; Springer Science+Business Media, Georgetown, Tex. New York, N.Y; 2006.

30. Kaneko K, Furusawa C. Consistency principle in biological dynamical systems. Theory in biosciences = Theorie in den Biowissenschaften. 2008;127(2):195-204. DOI: $10.1007 / \mathrm{s} 12064-008-0034-\mathrm{Z}$

31. Marquet PA, Quinones RA, Abades S, Labra F, Tognelli M, Arim M, Rivadeneira M. Scaling and power-laws in ecological systems. The Journal of Experimental Biology. 2005;208(Pt 9):1749-1769.

DOI: 10.1242/jeb.01588

32. Stauffer D. Phase transitions on fractals and networks. 2009;6783-6789.

DOI: 10.1007/978-0-387-30440-3_406

33. Nykter $M$, Price ND, Larjo A, Aho $T$, Kauffman SA, Yli-Harja O, Shmulevich I. Critical networks exhibit maximal information diversity in structure-dynamics relationships. Physical Review Letters. 2008;100(5):058702.

DOI: 10.1103/PhysRevLett.100.058702

34. Carlson JM, Doyle J. Complexity and robustness. Proceedings of the National Academy of Sciences of the United States of America. 2002;99(Suppl 1):2538-2545. DOI: $10.1073 /$ pnas.012582499

(c) 2019 Grinev et al.; This is an Open Access article distributed under the terms of the Creative Commons Attribution License (http://creativecommons.org/licenses/by/4.0), which permits unrestricted use, distribution, and reproduction in any medium, provided the original work is properly cited.

Peer-review history:

The peer review history for this paper can be accessed here: http://www.sdiarticle3.com/review-history/51032 